International Journal of Pure and Applied Mathematics

Volume 115 No. 1 2017, 59-66

ISSN: 1311-8080 (printed version); ISSN: 1314-3395 (on-line version)

url: http://www.ijpam.eu

doi: 10.12732/ijpam.v115i1.5

\title{
CERTAIN GENERATING FUNCTIONS OF GENERALISED HYPERGEOMETRIC 2D POLYNOMIALS FROM LIE-GROUP THEORETIC POINT OF VIEW
}

\author{
P.L. Rama Kameswari ${ }^{1 \S}$, V.S. Bhagavan ${ }^{2}$ \\ ${ }^{1,2}$ Department of Mathematics \\ K.L. University \\ Guntur Dt., A.P., INDIA \\ ${ }^{1}$ Department of Mathematics \\ Swarnandhra College of Engineering and Technology \\ Seetharampuram, Narsapuram-534 280 \\ West Godawari Dt., A.P., INDIA
}

\begin{abstract}
In this paper, we have obtained some novel generating functions of generalized hypergeometric $2 D$ polynomials $(G H 2 D P) U_{\mathrm{n}}(\beta ; \gamma ; x, y)$ by group theoretic method introduced by Louis Weisner.A suitable interpretation to the index $\mathrm{n}$ and parametres $\beta, \gamma$ are given,we introduced five linear partial differential operators wich they generate a Lie-algebra. Futher, we have derived the well known generating functions of Laguerre polynomialof two variables as an application.
\end{abstract}

AMS Subject Classification: 33C45, 33C47

Key Words: generalized hypergeometric 2D polynomials, generating functions

\section{Introduction}

Generating functions play a very vital role in the investigation of various useful properties of the functions which they generate. The name generating function was introduced by Laplace in 1812 and since then it has been developed into various directions and found wide applications in different branches of science

Received: January 4, 2017

Revised: $\quad$ April 12, 2017

Published: June 28, 2017

$\S$ Correspondence author
(C) 2017 Academic Publications, Ltd. url: www.acadpubl.eu 
and technology. A generating function may be used to define a set of functions, to determine recurrence relation or to evaluate certain integrals etc.

The object of the present paper is to derive some generating functions, which are to believed to be new, of Generalised Hypergeometric $2 D$ polynomials $(G H 2 D P)$, by suitably interpreting to the index $n$ and the parameters $\beta, \gamma$ simultaneously with the help of Weisner's group theoretic method. It may be mentioned that in the course of constructing a five dimensional Lie algebra we have obtained two operators such that, when operated on the polynomial under consideration, raise(lower) and lower(raise) the index and the parameter by one unit.

Many authors obtained generating functions for various orthogonal polynomials in the theory of special functions.

The generalized hypergeometric $2 D$ polynomials $(G H 2 D P) U_{n}(\beta ; \gamma ; x, y)$ satisfies the following ordinary differential equation

$$
\begin{aligned}
y(x-y) D^{2} U_{n}(x, y)-[(n+\beta-1) x-(\gamma+2 n-2) y] D U_{n}(x, y) & \\
& -n(\gamma+n-1) U_{n}(x, y)=0,
\end{aligned}
$$

where $D=\frac{d}{d y}$ and $U_{n}(x, y)=U_{n}(\beta ; \gamma ; x, y)$.

\section{Group Theoretic Discussion and Lie-Algebra}

In order to use Weisner's method, we construct from (1.1) the following partial differential equation by replacing $\frac{d}{d y}$ by $\frac{\partial}{\partial y}, n$ by $z \frac{\partial}{\partial z}, \beta$ by $t \frac{\partial}{\partial t}, \gamma$ by $s \frac{\partial}{\partial s}$ and $U_{n}(x, y)$ by $u(x, y, z, t, s)$ :

$$
\begin{aligned}
{\left[y(x-y) \frac{\partial^{2}}{\partial y^{2}}-z^{2} \frac{\partial^{2}}{\partial z^{2}}+\right.} & (2 y-x) z \frac{\partial^{2}}{\partial y \partial z}-t x \frac{\partial^{2}}{\partial y \partial t}+s y \frac{\partial^{2}}{\partial y \partial s} \\
& \left.-s z \frac{\partial^{2}}{\partial z \partial s}+(x-2 y) \frac{\partial}{\partial y}\right] u(x, y, z, t, s)=0,
\end{aligned}
$$

where

$$
u(x, y, z, t, s)=U_{n}(\beta ; \gamma ; x, y) z^{n} t^{\beta} s^{\gamma}
$$

is a solution of $(2.1)$, since $U_{n}(\beta ; \gamma ; x, y)$ is a solution of (1.1).

Now by following differential recurrence relations:

$$
D U_{n}(\beta ; \gamma ; x, y)=\frac{n}{y} U_{n}(\beta ; \gamma ; x, y)+\frac{n \beta x}{\gamma y} U_{n}-1(\beta+1 ; \gamma+1 ; x, y),
$$




$$
\begin{aligned}
D U_{n}(\beta ; \gamma ; x, y)= & \frac{(\beta-1) x-(\gamma+n-1) y}{y(x-y)} U_{n}(\beta ; \gamma ; x, y) \\
& +\frac{(\gamma-1)}{y(x-y)} U_{n+1}(\beta-1 ; \gamma-1 ; x, y)
\end{aligned}
$$

where $D=\frac{d}{d y}$.

Let us introduce a set of infinitesimal partial differential operators, $A_{i}, i=$ $1,2,3,4,5$ as follows:

$$
\begin{aligned}
& A_{1}=z \frac{\partial}{\partial z} \\
& A_{2}=t \frac{\partial}{\partial t} \\
& A_{3}=s \frac{\partial}{\partial s} \\
& A_{4}=\frac{y t s}{x z} \frac{\partial}{\partial y}-\frac{t s}{x} \frac{\partial}{\partial z}, \\
& A_{5}=\frac{z y(x-y)}{t s} \frac{\partial}{\partial y}+\frac{z^{2} y}{t s} \frac{\partial}{\partial z}-\frac{z x}{s} \frac{\partial}{\partial t}+\frac{y z}{t} \frac{\partial}{\partial s}-\frac{z(y-x)}{t s} .
\end{aligned}
$$

Then:

$$
\begin{aligned}
& A_{1}\left[z^{n} t^{\beta} s^{\gamma} U_{n}(\beta ; \gamma ; x, y)\right]=n z^{n} t^{\beta} s^{\gamma} U_{n}(\beta ; \gamma ; x, y), \\
& A_{2}\left[z^{n} t^{\beta} s^{\gamma} U_{n}(\beta ; \gamma ; x, y)\right]=\beta z^{n} t^{\beta} s^{\gamma} U_{n}(\beta ; \gamma ; x, y), \\
& A_{3}\left[z^{n} t^{\beta} s^{\gamma} U_{n}(\beta ; \gamma ; x, y)\right]=\gamma z^{n} t^{\beta} s^{\gamma} U_{n}(\beta ; \gamma ; x, y), \\
& A_{4}\left[z^{n} t^{\beta} s^{\gamma} U_{n}(\beta ; \gamma ; x, y)\right]=\frac{n \beta}{\gamma} z^{n-1} t^{\beta+1} s^{\gamma+1} U_{n-1}(\beta+1 ; \gamma+1 ; x, y), \\
& A_{5}\left[z^{n} t^{\beta} s^{\gamma} U_{n}(\beta ; \gamma ; x, y)\right]=(\gamma-1) z^{n+1} t^{\beta-1} s^{\gamma-1} U_{n+1}(\beta-1 ; \gamma-1 ; x, y) .
\end{aligned}
$$

We now proceed to find the commutator relations satisfied by $A_{i}, i=1,2,3,4,5$. Using the notation

$$
[A, B] u=(A B-B A) u,
$$

we receive the following commutator relations:

$$
\begin{aligned}
& {\left[A_{1}, A_{2}\right]=0, \quad\left[A_{2}, A_{3}\right]=0, \quad\left[A_{3}, A_{4}\right]=A_{4},} \\
& {\left[A_{1}, A_{3}\right]=0, \quad\left[A_{2}, A_{4}\right]=0, \quad\left[A_{3}, A_{5}\right]=-A_{5},} \\
& {\left[A_{1}, A_{4}\right]=-A_{4}, \quad\left[A_{2}, A_{5}\right]=-A_{5}, \quad\left[A_{3}, A_{4}\right]=\left(-A_{2}+A_{3}-1\right),} \\
& {\left[A_{1}, A_{5}\right]=A_{5} .}
\end{aligned}
$$


From the above commutator relations, we state the following theorem:

Theorem. The set of operators $A_{i}, i=1,2,3,4,5$ generates a five dimensional Lie algebra $L$.

It can be shown that the partial differential operator $L$, given by

$$
\begin{aligned}
L=y(x-y) \frac{\partial^{2}}{\partial y^{2}}-z^{2} \frac{\partial^{2}}{\partial z^{2}}+(2 y-x) z \frac{\partial^{2}}{\partial y \partial z}-t & \frac{\partial^{2}}{\partial y \partial t}+s y \frac{\partial^{2}}{\partial y \partial s} \\
& -s z \frac{\partial^{2}}{\partial z \partial s}+(x-2 y) \frac{\partial}{\partial y}
\end{aligned}
$$

can be expressed as follows:

$$
x^{-1} y L=A_{5} A_{4}+A_{1} A_{2} .
$$

It can easily verified that the operators $A_{i}, i=1,2,3,4,5$ commute with $x^{-1} y L$, i.e.

$$
\left[x^{-1} y L, A_{i}\right]=0, \quad i=1,2,3,4,5 .
$$

Therefore the extended form of the group generated by the each of the operators $A_{i}, i=1,2,3,4,5$. are

$$
\begin{aligned}
e^{a_{1} A_{1}} f(x, y, z, t, s)= & f\left(x, y, e^{a_{1}} z, t, s\right), \\
e^{a_{2} A_{2}} f(x, y, z, t, s)= & f\left(x, y, z, e^{a_{2}} t, s\right), \\
e^{a_{3} A_{3}} f(x, y, z, t, s)= & f\left(x, y, z, t, e^{a_{3}} s\right), \\
e^{a_{4} A_{4}} f(x, y, z, t, s)= & \left.f\left(x, \frac{x y z}{x z-a_{4} t s}, \frac{x z-a_{4} t s}{x}, t, s\right)\right), \\
e^{a_{5} A_{5}} f(x, y, z, t, s)= & \frac{t s}{(y-x) z a_{5}+s t} \\
& \times f\left(x, \frac{y s t}{(y-x) z a_{5}+s t}, \frac{z\left[(y-x) z a_{5}+s t\right]}{s t-x z a_{5}},\right. \\
& \left.\frac{s t-x z a_{5}}{s}, \frac{s\left[(y-x) z a_{5}+s t\right]}{s t-x z a_{5}}\right),
\end{aligned}
$$

where all $a_{i}(i=1,2,3,4,5)$ are arbitrary constants and $f(x, y, z, t, s)$ is arbitrary function.

Hence

$$
e^{a_{5} A_{5}} e^{a_{4} A_{4}} e^{a_{3} A_{3}} e^{a_{2} A_{2}} e^{a_{1} A_{1}} f(x, y, z, t, s)
$$




$$
=\frac{t s}{(y-x) z a_{5}+s t} f(x, \xi, \eta, \theta, \rho)
$$

where:

$$
\begin{aligned}
\xi & =\frac{x y z t s}{\left[(y-x) z_{a} 5+s t\right]\left[x z-a_{4}\left(s t-x z a_{5}\right)\right]}, \\
\eta & =\frac{e^{a_{1}}\left[(y-x) z_{a} 5+s t\right]\left[x z-a_{4}\left(s t-x z a_{5}\right)\right]}{x\left(s t-x z a_{5}\right)} \\
\theta & =\frac{e^{a_{2}} s t-x z a_{5}}{s} \\
\rho & =\frac{e^{a_{3}} s\left[(y-x) z_{a} 5+s t\right]}{\left(s t-x z a_{5}\right)}
\end{aligned}
$$

\section{Generating Functions}

We receive, from $(2.1)$, that $f(x, y, z, t, s)=z^{n} t^{\beta} s^{\gamma} U_{n}(\beta ; \gamma ; x, y)$ is a solution of the system:

$$
\begin{aligned}
& \left\{\begin{array} { l } 
{ L u = 0 } \\
{ ( A _ { 1 } - n ) u = 0 }
\end{array} \quad \left\{\begin{array} { l } 
{ L u = 0 } \\
{ ( A _ { 2 } - \beta ) u = 0 }
\end{array} \quad \left\{\begin{array}{l}
L u=0 \\
\left(A_{3}-\gamma\right) u=0
\end{array}\right.\right.\right. \\
& \left\{\begin{array}{l}
L u=0 \\
\left(A_{1}+A_{2}+A_{3}-n-\beta-\gamma\right) u=0
\end{array}\right.
\end{aligned}
$$

From (2.8), we receive

$$
S\left(x^{-1} y L\right)\left[z^{\nu} t^{\beta} s^{\gamma} U_{\nu}(\beta ; \gamma ; x, y)\right]=\left(x^{-1} y L\right) S\left[z^{\nu} t^{\beta} s^{\gamma} U_{\nu}(\beta ; \gamma ; x, y)\right],
$$

where

$$
S=e^{a_{5} A_{5}} e^{a_{4} A_{4}} e^{a_{3} A_{3}} e^{a_{2} A_{2}} e^{a_{1} A_{1}} .
$$

Hence the transformation $S\left[z^{\nu} t^{\beta} s^{\gamma} U_{\nu}(\beta ; \gamma ; x, y)\right]$ is also annulled by $\left(x^{-1} y L\right)$.

Choosing $a_{i}=0, i=1,2,3, a_{4}=b, a_{5}=c$, and writing $f(x, y, z, t, s)$ as $\left[z^{\nu} t^{\beta} s^{\gamma} U_{\nu}(\beta ; \gamma ; x, y)\right]$, i $\mathrm{n}(2.14)$, we obtain

$$
\begin{aligned}
e^{c A_{5}} e^{b A_{4}}\left[z^{\nu} t^{\beta} s^{\gamma} U_{\nu}(\beta ; \gamma ; x, y)\right]= & \frac{t s}{(y-x) z c+s t} \\
& \times\left[\frac{[(y-x) z c+s t][x z-b(s t-x z c)]}{[x z(s t-x z c)]}\right]^{\nu}
\end{aligned}
$$




$$
\begin{aligned}
& \times\left[1-\frac{x z c}{s t}\right]^{\beta}\left[\frac{(y-x) z c+s t}{(s t-x z c)}\right]^{\gamma} \\
& \times U_{\nu}\left[\beta ; \gamma ; x, \frac{x y z t s}{[(y-x) z c+s t][x z-b(s t-x z c)}\right] \\
& \times z^{\nu} t^{\beta} s^{\gamma} .
\end{aligned}
$$

On the other hand

$$
\begin{aligned}
e^{c A_{5}} e^{b A_{4}}\left[z^{\nu} t^{\beta} s^{\gamma} U_{\nu}(\beta ; \gamma ; x, y)\right]= & \sum_{l=0}^{\infty} \sum_{K=0}^{\infty} \frac{c^{k} A_{5}^{k}}{k !} \frac{b^{l} A_{4}^{l}}{l !}\left[U_{\nu}(\beta ; \gamma ; x, y) z^{\nu} t^{\beta} s^{\gamma}\right] \\
= & \sum_{l=0}^{\infty} \sum_{K=0}^{\infty} \frac{c^{k}}{k !} \frac{b^{l}}{l !}\left(\frac{\nu \beta}{\gamma}\right)_{l}(\gamma-1-l)_{k}\left(z^{-1} t s\right)^{l-k} \\
& \times\left[U_{\nu-l+k}(\beta+l-k ; \gamma+l-k ; x, y) z^{\nu} t^{\beta} s^{\gamma}\right] .
\end{aligned}
$$

Equating the expressios (3.1)and(3.2), we get

$$
\begin{aligned}
& \frac{t s}{(y-x) z c+s t}\left[\frac{[(y-x) z c+s t][x z-b(s t-x z c)]}{[x z(s t-x z c)]}\right]^{n} \\
& {\left[1-\frac{x z c}{s t}\right]^{\beta}\left[\frac{(y-x) z c+s t}{(s t-x z c)}\right]^{\gamma} } \\
& U_{n}\left[\beta ; \gamma ; x, \frac{x y z t s}{[(y-x) z c+s t][x z-b(s t-x z c)}\right] z^{n} t^{\beta} s^{\gamma} \\
= & \sum_{l=0}^{\infty} \sum_{K=0}^{\infty} \frac{c^{k}}{k !} \frac{b^{l}}{l !}\left(\frac{\nu \beta}{\gamma}\right)_{l}(\gamma-l)_{k}\left(z^{-1} t s\right)^{l-k} \\
& {\left[U_{\nu-l+k}(\beta+l-k ; \gamma+l-k ; x, y) z^{\nu} t^{\beta} s^{\gamma}\right] . }
\end{aligned}
$$

This may be regarded as a new generating relation which in turn yields a good number of particular new/known generating relationby attributing different values of $a_{i}, i=1,2,3,4,5$.

\section{Particular Cases}

1. when $b=1$ and $c=0$, replacing $z^{-1} t s$ by $w$ we have

$$
\left(\frac{x-w}{x}\right)^{\nu} U_{\nu}\left(\beta ; \gamma ; x, \frac{x y}{x-w}\right)
$$




$$
=\sum_{l=0}^{\infty} \frac{1}{l !}\left(\frac{\nu \beta}{\gamma}\right) U_{\nu-l}(\beta+l ; \gamma+l ; x, y) w^{l} .
$$

2. When $b=0$ and $c=1$, replacing $z t^{-1} s^{-1}$ by $w$, we get

$$
\begin{aligned}
{[1-(x-y) w]^{\nu+\gamma-1}(1-w x)^{\beta-\gamma-\nu} U_{\nu}\left(\beta ; \gamma ; x, \frac{y}{1-w(x-y)}\right) } & \\
= & \sum_{l=0}^{\infty} \frac{1}{k !}(\gamma)_{k} U_{\nu+k}(\beta-k ; \gamma-k ; x, y) w^{k}
\end{aligned}
$$

\section{Applications}

From the relations (3.1) and (3.2), we can derive the following generating functions for Lagaurre polynomials of two variables:

$$
\begin{gathered}
\sum_{l=0}^{\infty} \frac{(-1)^{l}}{l !} L_{\nu-l}^{(\alpha+l)}(x, y) w^{l}=L_{\nu}^{(\alpha)}(x+w, y) \\
\sum_{K=0}^{\infty} \frac{(-1)^{k}(1+\alpha)_{k}}{k !} L_{\nu+k}^{(\alpha-k)}(x, y) w^{k} \\
=(1+w(x-y))^{\alpha} \exp (-w x) L_{\nu}^{(\alpha)}[x+w x(x-y) ; y] .
\end{gathered}
$$

\section{References}

[1] A.K. Shukla and S.K. Meher, Group-theoretic origin of some generating functions for lagurre polynomials of two variables, App1. Math. Sci., 5, No. 61 (2011), 775-784.

[2] Asit Baran Chakrabarti, Group theoretic study of certain generalised functions of Jacobi polynomials, J. Indian Inst, Sci., 64(B) (1983), 97-103.

[3] E.D. Rainville, Special Functions, Macmillan Co., New York, 1960.

[4] E.B. Mc Bride, Obtaining Generating Functions, Springer, Verlag, New York, 1971.

[5] H.M. Srivastava, H.L. Manocha, A Treatise on Generating Functions, Halsted-Wiley, New York, 1984.

[6] I.K. Khanna, V.S. Bhagavan, Weisner's method to obtain generating relations for the generalised hypergeometric polynomial set, J. Phys. A: Gen and Math, 32 (1999), 989998. 
[7] I.K. Khanna, V.S. Bhagavan, Lie group theoretic origins of certain generating functions of the generalised hypergeometric polynomials, Integral Transforms Spec. Functions, 11, No. 2 (2001), 177-188.

[8] K.P. Samanta, B.C. Chandra, C.S. Bera, Some generating functions of modified gegenbauer polynomials by Lie algebraic method, Mathematics and Statistics, 2, No. 4 (2014), 172-178.

[9] L. Weisner, Group theoretic origins of certain generating functions, Pacific J. Math., 5 (1955), 1033-1039.

[10] M. Krosnicki, J. Czub, Gegenbaur polynomials in a theoretical study of the vibrational structure of system, Theoretical Chemistry Accounts, 115, No. 4 (2006), 322-329.

[11] Manic Chandra Mukherjee, Generating functions on Extended Jacobi polynomials from Lie group view point, Publications Matematiques, 40 (1996), 3-13.

[12] M.J.S. Shahwan, Lie theoretic generating relation of Laguerre 2D polynomials, Int. Jr. of Pure Applied Mathematics, 84, No. 2 (2013), 73-79.

[13] Subuhi Khan, Ghazale Yasmin, Lie theoretic generating relation of two variable Laguerre polynomials, Rep. math. phys., 51 (2003), 1-7.

[14] T. Srinivasulu, V.S. Bhagavan, Irreducible representation of $\operatorname{SL}(2, \mathrm{C})$ and generating relations for the generalized hypergeometric functions, Far East J. Math. Sci., 83, No. 2 (2013), 127-144.

[15] W. Miller Jr., Lie Theory and Special Functions, Academic Press, New York, 1968. 